\title{
IN SILICO ANALYSIS RELATED TO TIGR4 STRAIN IN STREPTOCOCCUS PNEUMONIAE
}

\author{
BALASANKAR KARAVADI*, POOJA SURESH
}

Department of Bioinformatics, School of Bio-Chemical Engineering, Sathyabama University, Chennai - 600 119, Tamil Nadu, India. Email: balasankarkaravadi@gmail.com

Received: 18 November 2017, Revised and Accepted: 19 December 2017

ABSTRACT

Objective: Numerous current investigations are done on the efficiency of natural components to combat the invasion by Streptococcus pneumoniae strain TIGR4; the main objective is to propose the most favorable ligand compound that could be effective to target the protein.

Methods: The normal segments from the Melissa officinalis are docked against serine/threonine protein kinase (STPK) receptor. The tools and programming utilized are modeler v 9.10 for displaying the protein structure, PubChem compound database to recover the synthetic structure of the ligands. ADMET was used to know the toxicity of the ligands and data warrior and the docking analysis was done by PyRx.

Result: The results show that 5-cedranone compounds satisfy the ADMET properties and are more favorable to bind with STPK receptor. The drug score of 5 -cedranone is 0.4572 and the $m$ binding energy is -7.9 .

Conclusions: The amino acid residue for the least binding energy for STPK is Ser 175 and Thr 167. Based on the ADMET analysis, 5-cedranone shows moderate cLogP and cLogS values and we predict 5-cedranone may not produce any side effects.

Keywords: Docking, ADMET, Modeler, Receptor, TIGR4.

(C) 2018 The Authors. Published by Innovare Academic Sciences Pvt Ltd. This is an open access article under the CC BY license (http://creativecommons. org/licenses/by/4. 0/) DOI: http://dx.doi.org/10.22159/ajpcr.2018.v11i4.23731

\section{INTRODUCTION}

Referred to later in 1886 as the pneumococcus for its part as a reason for pneumonia was first disengaged at the same time and freely by the U.S. Armed force doctor George Sternberg and the French scientific expert Louis Pasteur [1]. It was renamed Streptococcus pneumoniae in 1974 that it was fundamentally the same as streptococci [2]. S. pneumoniae assumed a focal part in showing that hereditary material comprises DNA. The living being was named Diplococcus pneumoniae from 1920 in view of its trademark appearance in Gram-recolored sputum. In 1928, Frederick Griffith exhibited change of life transforming innocuous pneumococcus into a deadly frame by coimmunizing the live pneumococci into a mouse alongside warm killed harmful pneumococci [3].

TIGR4 is an exceedingly harmful strain. Strains TIGR4 of S. pneumoniae is a clinical detach which was gotten from the blood test of 30-yearold male patient in Kongsvinger, Norway. TIGR4 was subjected different hereditary tests and it has been discovered that the strain is a harmful capsular strain of serotype 4. Genome of TIGR4 contains a grouping length of $2.34 \mathrm{Mb}$ which incorporates 2106 proteins and 2302 qualities with a GC substance of $39.7 \%$ [4].

In this article, we concentrate on the computational displaying of destructive proteins and approving the idea of the proteins as a future medication focus of TIGR4 in S. pneumoniae. They have likewise centered around distinguishing particular ligands for the previously mentioned proteins by utilizing the factual strategy for high throughput screening of lead atoms on the premise of structure action relationship. At long last, the lead atoms were approved utilizing ADMET descriptors [4,5]. The primary goal is to investigate the medication focus in TIGR4 strain of $S$. pneumoniae, subsequently helping in future medication improvement process. To demonstrate the unstructured protein utilizing homologous modeling - modeler, perform ADMET considers for the ligands chose from Lamiaceae family, recognize target protein of TIGR4 strain of Streptococcus pneumonia, distinguish the dynamic restricting site of the protein molecule, perform docking contemplated for the broke down protein receptor, and the ligands that fulfill the ADMET parameters, interpreting the association between the protein and the ligands $[6,7]$.

\section{METHODS}

The National Center for Biotechnology Information is a part of the United States National Library of Medicine, a branch of the National Institutes of Health and UniProt Knowledgebase is the focal center for the accumulation of utilitarian data on proteins databases were utilized to recover the objective proteins and template [8]. Basic local alignment search tool, a calculation was utilized for contrasting essential organic succession data of target and template [9]. PubChem is an electronic database which was utilized to recover data of the synthetic atoms and their exercises against natural examines [10]. Drug bank is a far-reaching database was utilized to recover data of the medications and their objectives [11]. Modeler is utilized for homology or similar demonstrating of protein three-dimensional (3D) structures. When we give an arrangement of a succession to be displayed with known related structures and modeler naturally computes a model containing all non-hydrogen molecules. Modeler actualizes similar protein structure demonstrating by fulfillment of spatial limitations and can perform numerous extra undertakings, including a new displaying of circles in protein structures, streamlining of different models of protein structure concerning an adaptably characterized target work, various arrangement of protein successions as well as structures, grouping, looking of arrangement databases, correlation of protein structures, and so forth [12]. PROCHECK was utilized to check stereochemical nature of a protein structure, creating various postscript plots investigating it is generally and buildup bydeposit geometry. It incorporates PROCHECK-NMR for checking the nature of structures illuminated by NMR, Ramachandran plot was utilized for it [13]. Pymol a perception programming was utilized for brilliant representation of biomolecules with 3D structure. This 
was exceptionally useful for discovering cooperation deposits amid docking ligand mixes with protein. BIOVIA discovery studio was likewise utilized for visualization, molecular docking process, energy minimization, and ADMET examination.

\section{RESULT AND DISCUSSION}

\section{Modeling and validation results}

The modeling was carried out for serine/threonine protein kinase (STPK) using homologous modeling shown in Fig. 1 and the validation of the modeled protein was done using SAVES, which showed $93.5 \%$ of residues in the favored region in Ramachandran plot as shown in Fig. 2 and Table 1 and evaluation of plot is given in Table 2 .

\section{ADMET}

The ADMET analysis for the resultant compounds was done. This provided the insight on drug likeness of the ligands, which is necessary to consider before designing of a drug. To use the compound for any

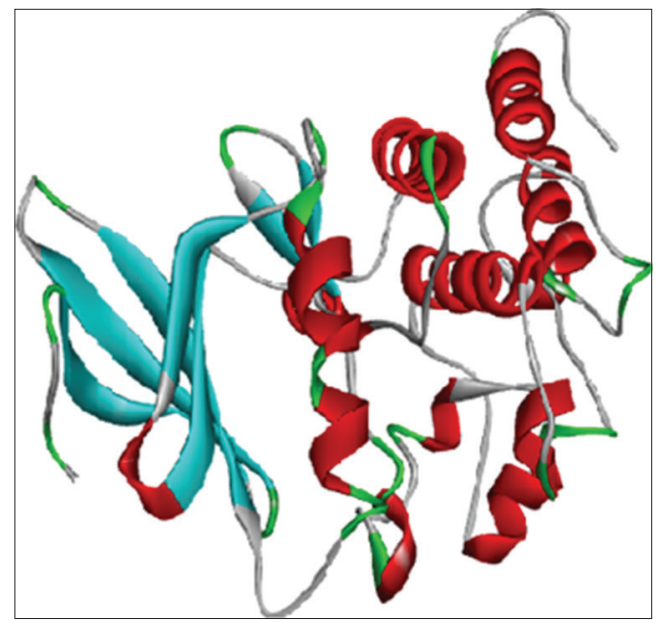

Fig. 1: Homologous modeling for Serine/threonine protein kinase

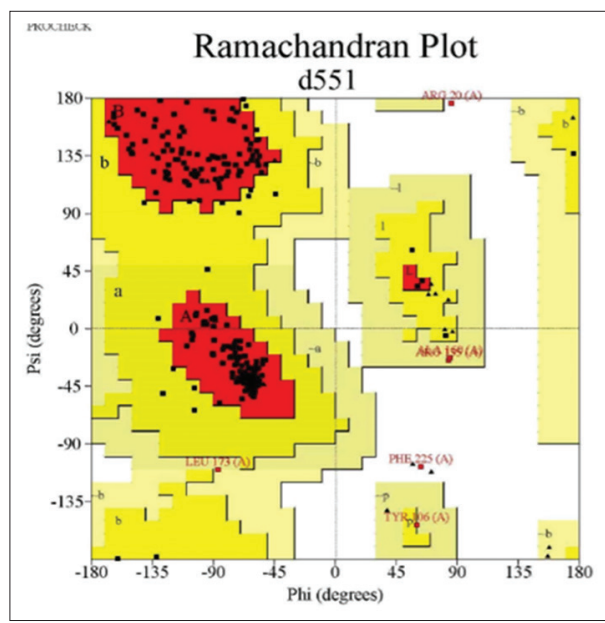

Fig. 2: Ramachandran plot for structure validation for serine/ threonine protein kinase further drug discovery, it should first get through to the toxicity test. Among the 17 ligands selected, one of the compounds (5-cedranone) did not showed any side effects such as tumorigenic, mutagenic, reproductive effective and irritant and the drug likeness and the drug score is also good. Thus, that compound was considered for further purpose.

\section{Docking results}

Various ligands were taken on the basis of their action on the variants of the protein. The 3D structure of the ligands was obtained from PubChem. The 3D ligands were docked with the modeled structure of the protein and their affectivity was seen on the basis of their binding energy as shown in Table 3. The binding site for the modeled protein was Ser 175 and Thr 167 amino acid residue. The distance between the atoms which are involved in $\mathrm{H}$-bond is 3.08 and 3.16. Effective results were obtained using the PyRx v 8.0 software which provided the ligand that can be taken for further study on drug discovery; 5-cedranone shows the binding energy among the ligands and its interaction details as shown in Table 4.

\section{D interaction of the receptor with the ligand}

The hydrophobic interaction of 5-cedranone with STPK was shown in Ser 175 and Thr 167 amino acid. The bond distance between the atoms which are involved in hydrogen bond is 3.16 and 3.08; they are denoted with the green dotted line. $\mathrm{C}, \mathrm{N}$, and $\mathrm{O}$ are the main chain. $\mathrm{C1}^{\prime}-\mathrm{C}^{\prime}{ }^{\prime}=1^{\prime}-5^{\prime}$

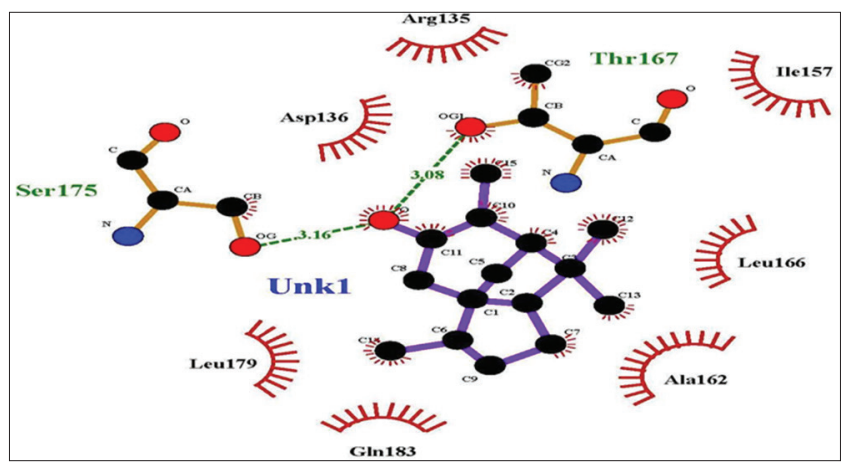

Fig. 3: Hydrophobic interaction of 5-cedranone with serine/ threonine protein kinase

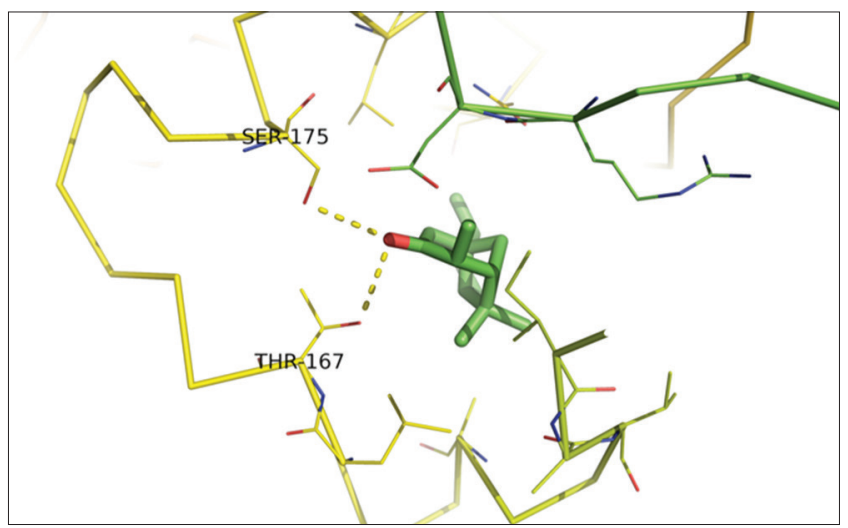

Fig. 4: Three-dimensional representation of the interaction between 5 -cedranone with serine/threonine protein kinase

Table 1: Target and template description

\begin{tabular}{lllllll}
\hline Target protein & Length & Template & Length & Similarity (\%) & & \multicolumn{2}{c}{ Ramachandran plot (\%) } & Outliers & Favored & Allowed \\
\hline STPK (Q97PA9) & & & & & & \\
\hline
\end{tabular}

STPK: Serine/threonine protein kinase 
is the pentose carbons and CG is the carbon gamma in $3^{\text {rd }}$ position. The red circle indicates oxygen, dark color circle indicates carbon, and blue indicates nitrogen atoms are as shown in Fig. 3.

\section{D representation of the receptor with the ligand}

The 3D representation was done using Biovia visualizer. The image is been displayed in stick format. The dotted lines are the distances between atoms which are involved in hydrogen bond are as shown in Fig. 4.

4EQM were found to be the suitable template for modeling the membrane protein (Sp_Q97PA9) with a sequence identity of $48 \%$, respectively. The modeled protein has about (93.5\%) of residues in the most favored region of the Ramachandran plot. Literature search for ligands from Lamiaceae family, particularly for Melissa officinalis (lemon balm) plants was chosen, and the structure of the ligands was retrieved from PubChem. The 17 ligands were docked with the modeled proteins structure, respectively, and the dock score is calculated by PyRx v8.0 software suite. Among various ligands, best high scoring compounds were considered to be the drug candidate. After docking, the ligands were further subjected to ADMET analysis. In ADMET analysis, among the 17 ligands selected, one of the compounds (5-cedranone) did not showed any side effects such as tumorigenic, mutagenic, reproductive effective, irritant and the drug likeness and the drug score is also good. Thus, that compound can be considered for further purpose. Homology modeling was performed for membrane protein (Sp_Q97PA9) using the respective template structure $(4 \mathrm{EQM})$, and finally, modeled protein was validated through SAVS and $93.5 \%$ residues in target proteins are present in the allowed region of Ramachandran plot. ADMET properties for the ligands having better binding energy and maximum interaction with the active site residues were analyzed. Based on our analysis, it has been found that the ligands which had maximum dock score have proper cLogP and cLogS values shown 5-cedranone was the best ligand for the modeled protein based.

\section{CONCLUSION}

The current study focused on molecular docking analysis of the ligand compound with the protein STPK receptor nucleotide binding protein. The main goal was to propose the most favorable ligand compound that could be effective to target the protein. From the results, it was determined that 5-cedranone formed stable complex compared to

Table 2: Evaluation of residues

\begin{tabular}{llll}
\hline Residue & A 15:VAL & $-127.32,-50.57$ & \\
Residue & A 54:ALA & $-103.97,-63.70$ & Allowed region \\
Residue & A 83:ASP & $81.54,-5.28$ & Allowed region \\
Residue & A 135:ARG & $83.69,-24.66$ & Allowed region \\
Residue & A 145:THR & $-109.60,176.75$ & Allowed region \\
Residue & A 158:ALA & $-63.98,103.49$ & Allowed region \\
Residue & A 160:ALA & $84.40,-22.72$ & Allowed region \\
Residue & A 167:THR & $-70.98,90.42$ & Allowed region \\
Residue & A 169:THR & $-44.04,105.16$ & Allowed region \\
Residue & A 211:PRO & $-94.22,46.19$ & Allowed region \\
Residue & A 225:PHE & $63.14,-107.70$ & Allowed region \\
Residue & A 231:SER & $-62.22,108.09$ & $175.61,136.59$ \\
Residue & A 236:ASN & $-67.78,179.06$ & Allowed region \\
Residue & A 258:ARG & $85.69,176.05$ & Allowed region \\
Residue & A 20:ARG & $60.13,-153.42$ & Outlier region \\
Residue & A 106:TYR & $-86.59,-110.26$ \\
Residue & A 173:LEU & $-100.29,100.01$ \\
Residue & A 237:PRO & Outlier region \\
\hline
\end{tabular}

Number of residues in favored region ( 98.0\% expected): 258 (93.5\%), number of residues in allowed region ( 2.0\% expected): 14 (5.1\%), number of residues in outlier region: $4(1.4 \%)$

Table 3: Docking results of 17 ligands showing binding energy for STPK (Model)

\begin{tabular}{ll}
\hline Ligands & binding energy \\
\hline (-)-Globulol (12304985) & -7.3 \\
(E)-beta-ocimene (5281553) & -5.3 \\
(Z)-BETA-OCIMENE (5320250) & -6.2 \\
5-cedranone (6430324) & -7.9 \\
BETA-CARYOPHYLLENE (5281515) & -6.3 \\
CARVACROL (10364) & -6.3 \\
Caryophylene_oxide (14350) & -5.9 \\
CITRONELLAL (7794) & -5.4 \\
Citronellyl_formate (7778) & -6 \\
GERANIAL (638011) & -5.5 \\
GERANIOL (637566) & -6.3 \\
GERANYL ACETATE (1549026) & -5.5 \\
Thymol (6989) & -5.8 \\
Germacrene D (5373727) & -7.6 \\
NERAL (643779) & -5.5 \\
\hline
\end{tabular}

STPK: Serine/threonine protein kinase

Table 4: Amino acid residue for least binding energy for STPK

\begin{tabular}{llllll}
\hline Ligands & Target & Binding energy Kcal/mol & Amino acid residue & Binding affinity & Autodock elements of ligands \\
\hline 5-cedranone & STPK & -7.9 & Ser 175, Thr 167 & -7.9 & C OA \\
\hline
\end{tabular}

STPK: Serine/threonine protein kinase 
other compounds with their respective receptor. This compound showed maximum binding affinity to the receptor. These could be the best compound to strain TIGR4 S. pneumoniae. ADMET properties were also analyzed so these can be accepted as potential therapeutic for strain TIGR4 S. pneumoniae. Based on the ADMET analysis, 5-cedranone shows moderate cLogP and cLogS values and we predict 5-cedranone may not produce any side effects such as tumorigenic, mutagenic, reproductive effective and irritant, the drug likeness and drug score is also good. In docking, 5-cedranone also binds with STPK with least energy value. Hence, we conclude that the 5-cedranone might be act as a good anti-S. pneumoniae (TIGR4) drug. Further, wet laboratory analysis may be needed to confirm this drug action.

\section{REFERENCES}

1. Plotkin SA, Orenstein W, Offit PA. Vaccines. Philadelphia, PA: Elsevier - Saunders; 2012. p. 542-51.

2. Wainer H. Medical Illuminations; Using Evidence, Visualization and Statistical Thinking to Improve Healthcare. Oxford: Oxford University Press; 2014. p. 53-4.

3. Griffith F. The Significance of Pneumococcal Types. J Hyg 1928;27:113-59.

4. Karavadi B, Suresh MX. Homology modeling and molecular drug design approach in identifying drug targets of TIGR4 in Streptococcus pneumonia. Biosci Biotechnol Res Asia 2014;11:517-22.

5. Karavadi B, Suresh XM. Homology modeling of polymerase and CPS biosynthesis proteins in CGSP14 strain of Streptococcus pneumonia and its ligand identification: An in silico approach. Asian J Pharm Clin Res 2014;7:162-5.

6. Karavadi B, Suresh MX. In silico modeling of capsular polysaccharide biosynthesis protein and tyrosine kinase of G54 strain in Streptococcus pneumoniae and their ligand identification. Int $\mathrm{J}$ Pharm Pharm Sci 2014;6:547-50.

7. Karavadi B, Suresh MX. Receptor identification and lead molecular discovery of phage encoded protein in TCH8431/19A strain of Streptococcus pneumoniae: A computational approach. Int J Appl Pharm 2014;6:6-10.

8. Pundir S, Martin MJ, O'Donovan C, UniProt Consortium. UniProt tools. Curr Protoc Bioinformatics 2016;53:1.29.1-15.

9. Altschul SF, Gish W, Miller W, Myers EW, Lipman DJ. Basic local alignment search tool. J Mol Biol 1990;215:403-10.

10. Kim S, Thiessen PA, Bolton EE, Chen J, Fu G, Gindulyte A. PubChem substance and compound databases. Nucleic Acids Res 2016;44:1202-13.

11. Wishart DS, Knox C, Guo AC, Shrivastava S, Hassanali M, Stothard P, et al. Drug bank: A comprehensive resource for in silico drug discovery and exploration. Nucleic Acids Res 2006;34:668-72.

12. Spill YG, Kim SJ, Schneidman-Duhovny D, Russel D, Webb B, Sali A, et al. SAXS merge: An automated statistical method to merge SAXS profiles using Gaussian processes. J Synchrotron Radiat 2014;21:203-8.

13. Ramachandran GN, Ramakrishnan C, Sasisekharan V. Stereochemistry of polypeptide chain configurations. J Mol Biol 1963;7:95-9. 